\title{
Introduction to the Project
}

\section{₹ [DOES-NOT-EQUAL]}

This project is about disentanglement. It is a theoretical hypothesis disentangling the phenomenon of pregnancy from the phenomenon of childbearing, supported by a narrative of personal experience as best as I could recall it. When you have been raised and groomed to believe that pregnancy is equivalent to - if not also inherently entailing-the phenomena of labor, childbirth, and motherhood wrapped up in a mythos of unconditional love and desire, anything short of these expectations of equivalence becomes a site of harm and humiliation. This project is also intended to be an outright condemnation of our shame and blame culture, especially when it is a gendered phenomenon-an unnecessary bearing-down on women and girls for no other reason than their anatomical differences from men.

I would like to identify this project as a feminist phenomenological project-at least in method-yet, ultimately, it is experimental as to what this project might yield. Philosophical analysis is not usually traditional fare for feminism and feminist critique; few philosophers deal in-much less construct narrative in - the form of the graphic novel. ${ }^{1}$ That said, much good, feminist work has already been done through the medium of the graphic novel with which I will not be able to compete but, hopefully, only complement. To this point, I place this work among other feminist theorists and philosophers breaking the boundaries down regarding "what counts" for the work of philosophy. 
When I became aware of the \#shoutyourabortion Twitter hashtag, I was hit by a wave of conflicting feelings as latent memories resurfaced, and yet this provocation also came with a kind of clarity. ${ }^{2} \mathrm{Up}$ to that point I had an uncritical but functional distinction embedded in my recall of events: although I had experienced a spontaneous miscarriage in my first pregnancy, I had also classified in my mind my third pregnancy as a miscarriage when in fact I had undergone an abortive procedure in what could be considered an abortion clinic. I had-and my family had as well-classified it in this way because this had been a wanted pregnancy and I had it aborted in order to- by all accountssave my life. What came clearly into view with the \#shoutyourabortion thread was that this experience of pregnancy counted as an abortion. The public knowledge or "shouting" of this would mean that I could not hide behind the safety of the "less shameful" reasons for having an abortion. Just because I did not seek it out and had expected my "baby" did not mean that I could maintain distinctions I had comfortably kept any longer. Intellectually - and, to a degree, emotionally-there had been no demand that I grapple with the ambiguity of my situation. As Simone de Beauvoir states it in The Ethics of Ambiguity (1947, part II, $\S 5, \uparrow 10$ ), when an "individual lives in a situation of falsehood, the falsehood is violence, tyranny: shall I tell the truth in order to free the victim? It would first be necessary to create a situation of such a kind that the truth might be bearable and that, though losing his [or her] illusions, the deluded individual might again find about him [or her] reasons for hoping." This project is a hopeful one in its negative assessment of pregnancy and in the disentanglement of pregnancy from the bearing of children; it is motivated by a coming-to-terms with falsehoods and illusions that most pregnant women have come to bear. In this project, in grappling with the truth of what had been "my situation," I hope to provide what has been up to now an underdeveloped point of solidarity among women: that, once pregnant, there is a phenomenon of undergoing that is temporal and substantial and of which we will all find ourselves postpartum. Most important to this point of possible solidarity, this is an undergoing given to all pregnancy whether or not a child is born.

As Sandra Bartky $(1990,97)$ puts it, "Moral psychology has told us a story, but not the whole story," which prompts me toward the necessary unpacking of the conditions of shame and guilt that come with the per- 
ceived failure(s) of a "pregnancy that does not lead to the bearing of a child" set in this project and in cooperation with her phenomenology of oppression; for this project, shame and guilt are not "moral emotions." With a project like this, I am imagining how the conditions might be set such that "the truth might be bearable" and I may reevaluate the oppressive and victimizing demands of a misogynist society, starting with my own illusions. This project is an attempt to deinternalize the shame and grief of a compartmentalized and suppressed memory. This is the significance of part I's title, "Miscarriage or Abortion?" as my way of \#shoutingmyabortion, as well as the significance of the title of part IV, "Griefwork," as I found myself needing to ask the question: How do you get over what you cannot get over?

\section{HAVING (AND NOT HAVING) "A BABY"}

My story-the content of the graphic novel-is thick with cisgendered white privilege, especially as it begins with my introduction into pregnant naïveté. In 2006, I was young, married, and employed, and therefore it came time in the script to "start a family." Instead, I was initiated into a quiet community of women who knew how naive and privileged-and damning - that assumption can be. I ran up against a razor's edge where I had assumed there would be nothing but a cushion of joy and accomplishment. This is what I had described to myself as "a privilege that is not a privilege": it is a privilege to embody the political, gender, and social norms of being pregnant. I know there are many women for whom getting pregnant would be a privilege, and one they have been denied. Yet pregnancy without its childbearing function, as with "pregnancy loss," from privilege. Still, there was more to be had in this privilege that is not a privilege: I found many other women who knew this contradiction in its painful intimacy ${ }^{4}$ - to have been pregnant without the production of a child-such that this, too, became its own, new form of epistemic privilege.

In "Being Torn: Toward a Phenomenology of Unwanted Pregnancy," Caroline Lundquist $(2008,136)$ calls for a more inclusive phenomenology of pregnancy that "gives voice to the multitudes of women who have not chosen their pregnancies," and this project follows that call. 
At the same time, while deviating from Lundquist's project, I will bracket desire and choice regarding pregnancy in my phenomenological approach. Lundquist accounted for phenomena attached to the rejected and denied pregnancy; I suspend that phenomenon to get at the quality of expellation ${ }^{5}$ given to all pregnancy. That my project is negative toward pregnancy can still be appropriately contextualized in her call for an inclusive phenomenology, as I will argue it, especially as I outline what I call a death-within-the-self-the phenomenal expulsion of expectation - as an existential given to all pregnancy. Lundquist specifically states that "until women have the vocabulary with which to express ambivalent and even negative feelings regarding pregnancies ... they will continue to suffer in silence. By offering a more inclusive account of pregnancy, feminist phenomenology has the potential to produce such a vocabulary. The lived experience of pregnancy is so radically diverse, and so heavily conditioned, that it behooves feminist philosophers to continue to explore it" (152). What this project opens up to examination is the ontological fact that all pregnancies are open to miscarriage, so I also ask: Can't there be grief in birth? Doesn't all postpartum experience share in the experience of miscarriage in a deep and fundamental way?

Added to this project, with this expellation, at least ontologically, grief is on the scene. I will argue this grief as phenomenologically on the scene as a necessary labor that cannot be glossed over and gotten over-calling this necessary labor a griefwork that needs to be done and ought not be done by women alone. Although I experience grief alone in that it alienates me from others, it is not mine and this grief ought not leave me to myself. The idea of griefwork outlined here is not captured in the private, personal exercise of grieving, because the more I allow my grief and "do it," the more, in my alienation, I am isolated by it.

It has become the case that "the foetus is integral to their [and her] procreative aims. It is valuable to them because having a child is an important desire of theirs" (Porter 2015, 66). This ontic assumption of childbearing is another kind of alienation from meaningful possibilities, because all meaning of pregnant embodiment rests on the production of a child. Here is where I find the disconnect (the razor's edge) embedded in the promotion of the "successful pregnancy," such that even reproduction as a production renders the force of her need to be a productive (read: valuable) member of society. Yet when pregnancy 
leads to normative outcomes of childbearing, at the same time, any alternative to this expectation of outcome is rendered unthinkable and becomes read as a most undesirable situation, fundamentally "unspoken." Analogous to the alienation found in wage work, the productive pregnancy can alienate me from my individual power for collective action-finding myself as a woman among women in having been pregnant-now isolated each from the other. Similarly, in the way that one might think having a job gives one status and protects social standing, it is an artificially induced and manufactured division from those unemployed (yet the harder I work, the more I might desire the alienation than the overcoming of it; I believe myself to have found myself in the productivity).

Hilde Lindemann $(2015,80)$ acknowledges this as a kind of "plot" and states that "master narratives ... serve as hermeneutical resources" and the bearing of the child becomes integral to the story: "The baby is wanted." She notes how "many aspects [of her pregnancy] are [now] purposeful," by "calling the fetus into personhood" (82-83). Iris Marion Young (2005) writes that one of the most important instruments to the advancement of the entanglement--the "plot"-of childbearing with pregnancy is the ultrasound image. ${ }^{6}$ It is often the catalyst for the wanted pregnancy, a sign of the fetus, but also part of the setup in which women can fail and in which women may come to grieve. This is why I cannot attend to grief psychologically because I provide no therapeutic relief in this project: I only describe what labor and work must now be required, what leads to its onset and its sustenance. I will defend the idea that grief must be grappled with, as labor-intensive, or else it further entangles us in the plot of pregnant productivity.

I will add to this reading an active critique of the idea that the bearing of children is an "accomplishment" because it is attached to the master narrative of neoliberal ideology. Pregnancy, whichever way it goes, when in the function of a misogynist and neoliberal culture, with its corresponding mechanisms and affectations, is a trap, a setup. The failed or unwanted pregnancy becomes a "woman problem," or, worse, "her fault." So here I plan to shut that idea down. Maybe the outcome of this, as it is an experimental project, will be that we do not continue to be divided by paternal and neoliberal interests to play our part in the master narrative of productive pregnancy; perhaps instead solidarity will be found with the woman who has miscarried, as she might recog- 
nize herself in the woman who has aborted her pregnancy, and again each with the woman who has "successfully" given birth.

\section{NOTES}

1. It is fairly recently that the first academic dissertation was delivered as a graphic novel. See Sousanis 2015.

2. Hardy and Kukla $(2015,109)$ describe this connection better than I do: "The Internet provides an unprecedented and dizzying source of communities united by common experiences, as well as equally unprecedented tools for creating new communities. Women are using these new resources to find ways of getting uptake for and giving shape to narratives that were previously resistant to articulation."

3. I note this in quotes because pregnancy loss is code for miscarriage and stillbirth, but in this project I will argue that all pregnancies lead to a kind of loss - an emptying out of the situation.

4. To this point, Lindemann $(2015,89)$ states that sometimes we must "leave unspoken the stories that are too painful for her to tell. Some sorrows go too deep for words, and others are too fragmentary or chaotic or fleeting to be captured by words."

5. I call it expellation because of the additional quality to pregnancy superseding the physiological "expulsion" given to all pregnancy and how medicine might define the phenomena of pregnancy and "postpartum." Expellation here includes the phenomenological content of possibility and expectation that comes along with the event of pregnancy termination and independent of fetal status.

6. In her 2003 postscript to "Pregnant Embodiment: Subjectivity and Alienation" discussed in part III (and published in Young 2005). 